\title{
Tenotomia do tendão flexor profundo no tratamento da deformidade flexural adquirida na articulação interfalangeana distal em um equino
}

\author{
Tenotomy of the deep digital flexor tendon in the treatment of an acquired flexural deformity \\ of the distal interphalangeal joint of a horse \\ Luciana Paula Merini', Carlos Afonso de Castro Beck ${ }^{2}$, Alice Guigno Gomes ${ }^{3}$, \\ Graziela Tizoni Cescon ${ }^{3}$, Cassius Alexandre Ramos ${ }^{3}$, Roberta Bergamin Scarton ${ }^{3}$, \\ Maicon Bonini Faria ${ }^{3} \&$ Samuel Carnesella ${ }^{3}$
}

\begin{abstract}
RESUMO
As deformidades flexurais são frequentemente vistas em animais recém-nascidos ou desenvolvem-se entre os dois primeiros anos de vida, porém, nos cavalos podem ser adquiridas em qualquer idade. Podem ser uni ou bilaterais, e geralmente se produzem como deformações flexoras das articulações interfalangeanas distais e metacarpofalangeanas. As causas relacionadas são as nutricionais, dolorosas, traumáticas, bem como a predisposição para o rápido crescimento. Foi atendido um equino, macho, castrado, sem raça definida, com oito anos de idade, $260 \mathrm{~kg}$, utilizado para tração, que apresentava uma deformação flexora da articulação interfalangeana distal de grau II do membro torácico esquerdo. Pressupondo que as principais causas tenham sido a traumática e a dolorosa, foi estipulado como tratamento a tenotomia do tendão flexor digital profundo. O pós-cirúrgico constou do uso de anti-inflamatório não esteroidal, casqueamento corretivo e sessões de fisioterapia diárias. Este trabalho mostra com êxito a utilização da tenotomia do tendão flexor digital profundo para correção da deformação flexural adquirida na articulação interfalangeana distal unilateral em um equino adulto. Com o tratamento estipulado, o membro locomotor do animal retornou à sua conformação normal e após seis meses não houve sinal de recidiva, o que garantiu uma melhora significativa na sua qualidade de vida.
\end{abstract}

Descritores: contratura tendínea, deformação flexural, tenotomia, equino.

\begin{abstract}
Flexural deformities are usually found in foal at birth or develop in the first two years of life but may be acquired at any age. They can be uni and bilateral, and generally are produced as flexural deformity of the distal interphalangeal and metacarpophalangeal joints. The related causes are nutritional, pain, trauma and the predisposition for fast growth. An eight-yearold gelded horse, with no defined breed, weighing $260 \mathrm{~kg}$, used for animal traction presented a flexural deformity of the distal interphalangeal joint of the left thoracic member of stage II contractural deformities, having as possible causes trauma and pain. The tenotomy of the deep digital flexor tendon was stipulated as treatment. The postoperative consisted of the nonsteroidal antiinflammatory, corrective shoeing and daily sessions of physiotherapy. The present work shows with success the use of the tenotomy of the deep digital flexor tendon for correction of the acquired flexural deformation in the unilateral distal interphalangeal joint in an adult equine. With the stipulated treatment the animal returned to its normal configuration of the member and after six months it did not have any sign of recurrence, what guaranteed a significant improvement in its quality of life.
\end{abstract}

Keywords: tendon contracture, flexural deformity, tenotomy, horse.

${ }^{1}$ Programa de Residência em Medicina Veterinária, Hospital de Clínicas Veterinárias (HCV), Universidade Federal do Rio Grande do Sul (UFRGS), Av. Bento Gonçalves no. 9090, Bairro Agronomia, CEP 91540-000 Porto Alegre, RS, Brasil. 'Departamento de Medicina Animal, Faculdade de Veterinária (FaVet), UFRGS. ${ }^{3}$ Graduação, Medicina Veterinária, FaVet, UFRGS. CORRESPONDÊNCIA: L.P. Merini [lulyvet@yahoo.com.br - Fax: + 55 (51) 3308 6112]. 


\section{INTRODUÇÃO}

As deformações flexoras adquiridas podem ser uni ou bilaterais, e geralmente localizam-se nas articulações interfalangeanas distais e metacarpofalangeanas [12]. As causas frequentes desta afecção em potros são as nutricionais, a predisposição genética e a restrição do exercício, enquanto que nos animais adultos a mesma está relacionada com a dor e o trauma [3,7,8].

A deformação flexora da articulação interfalangeana distal, também denominada contratura do tendão flexor digital profundo (TFDP), manifesta-se clinicamente com a elevação dos talões e aspecto de "casco em pinça" [12].

Com o propósito de prognóstico e avaliação do tratamento, a deformação da articulação interfalangeana distal está dividida em estágios I e II. O estágio I da contração ocorre quando o ângulo entre a parede dorsal do casco e o solo não ultrapassa $90^{\circ}$, enquanto que no estágio II de contração, a parede dorsal do casco está aquém da vertical $\left(>90^{\circ}\right)$ [2]. Animais que apresentam o estágio I da deformação devem ser tratados mediante a desmotomia do ligamento frenador carpiano (inferior). A tenotomia do (TFDP) está indicada nos casos graves e crônicos de contratura do flexor digital profundo (estágio II) [4,7].

A intervenção cirúrgica está indicada nos casos que não respondem ao tratamento conservador. A cirurgia imediata é oportuna naqueles casos em que se necessita de rápida correção da deformação para evitar o desenvolvimento de alterações articulares degenerativas permanentes [8].

O objetivo deste trabalho é demonstrar a utilização da tenotomia do TFDP para a correção da deformação flexural adquirida na articulação interfalangeana distal unilateral em um equino adulto.

\section{RELATO DE CASO}

Foi encaminhado para o Setor de Clínica de Grandes Animais do Hospital de Clínicas Veterinárias (HCV) da Universidade Federal do Rio Grande do Sul (UFRGS), um equino macho castrado, sem raça definida, com oito anos de idade, $260 \mathrm{~kg}$, utilizado para tração.

O animal apresentava uma deformação flexora da articulação interfalangeana distal de grau II no membro anterior esquerdo (MAE) (Figura 1A). Na inspeção, notou-se uma conformação anormal do membro com consequente desgaste da parede do casco. Na avaliação radiográfica, não foram observadas alterações ósseas e articulares.

Foi estipulado como tratamento a tenotomia do TFDP. O procedimento cirúrgico foi realizado com o animal em estação. Para sedação, foi utilizado cloridrato de xilazina ${ }^{1}\left(1 \mathrm{mg} \cdot \mathrm{kg}^{-1}\right)$, intravenoso (IV). Foi preparado assepticamente o terço médio lateral do metatarso esquerdo e realizado bloqueio local da área de incisão com $10 \mathrm{~mL}$ de cloridrato de lidocaína ${ }^{2} 2 \%$ sem vaso constritor. Após incisão de, aproximadamente, dez centímetros de pele, foi realizado divulsão romba do tecido subcutâneo. Localizou-se o TFDP e dissecou-se este do osso metacarpiano e do tendão flexor digital superficial, possibilitando assim a sua exteriorização. Foi infiltrado $5 \mathrm{~mL}$ de cloridrato de lidocaína $2 \%$ sem vaso constritor no local da secção do tendão sendo este seccionado com bisturi após 10 minutos de intervalo (Figura 1B). Para redução de espaço morto e sutura de pele, utilizaram-se métodos cirúrgicos rotineiros.

O tratamento pós-operatório constou de: fenilbutazona ${ }^{3}\left(20 \mathrm{mg} / \mathrm{kg}^{-1}\right)$, IV, SID, durante 10 dias, limpeza da ferida, atadura compressiva e repouso. $\mathrm{O}$ casqueamento corretivo do membro se deu com três dias de pós-operatório e possibilitou ao membro o retorno à sua conformação normal (Figura $1 \mathrm{C}$ ). A retirada dos pontos se deu com dez dias de pós-operatório e a partir do $11^{\circ}$ até o $30^{\circ}$ dia foram realizadas sessões diárias de fisioterapia que constavam de caminhadas ao passo de 15 minutos seguidos de dez passos em marcha ré. Não houve complicações cirúrgicas e o animal recebeu alta após o tratamento estipulado. $\mathrm{O}$ animal foi reavaliado após seis meses e não apresentava nenhum sinal de recidiva.

\section{DISCUSSÃO}

As deformações flexoras adquiridas podem ocorrer em qualquer idade [6] e ser uni ou bilaterais, sendo que geralmente se produzem como deformações flexoras das articulações interfalangeanas distais e metacarpofalangeanas [12]. O caso em questão tratou-se de uma deformação flexora adquirida unilateral da articulação interfalangeana distal.

A patogênese das deformações flexoras adquiridas em animais adultos se relaciona frequentemente com a dor e o trauma sobre o tendão, o que leva à contração tendínea pela formação de tecido cicatricial fibroso. Qualquer condição de dor pode ser responsável pela flexão como sinal de reflexo de proteção e 

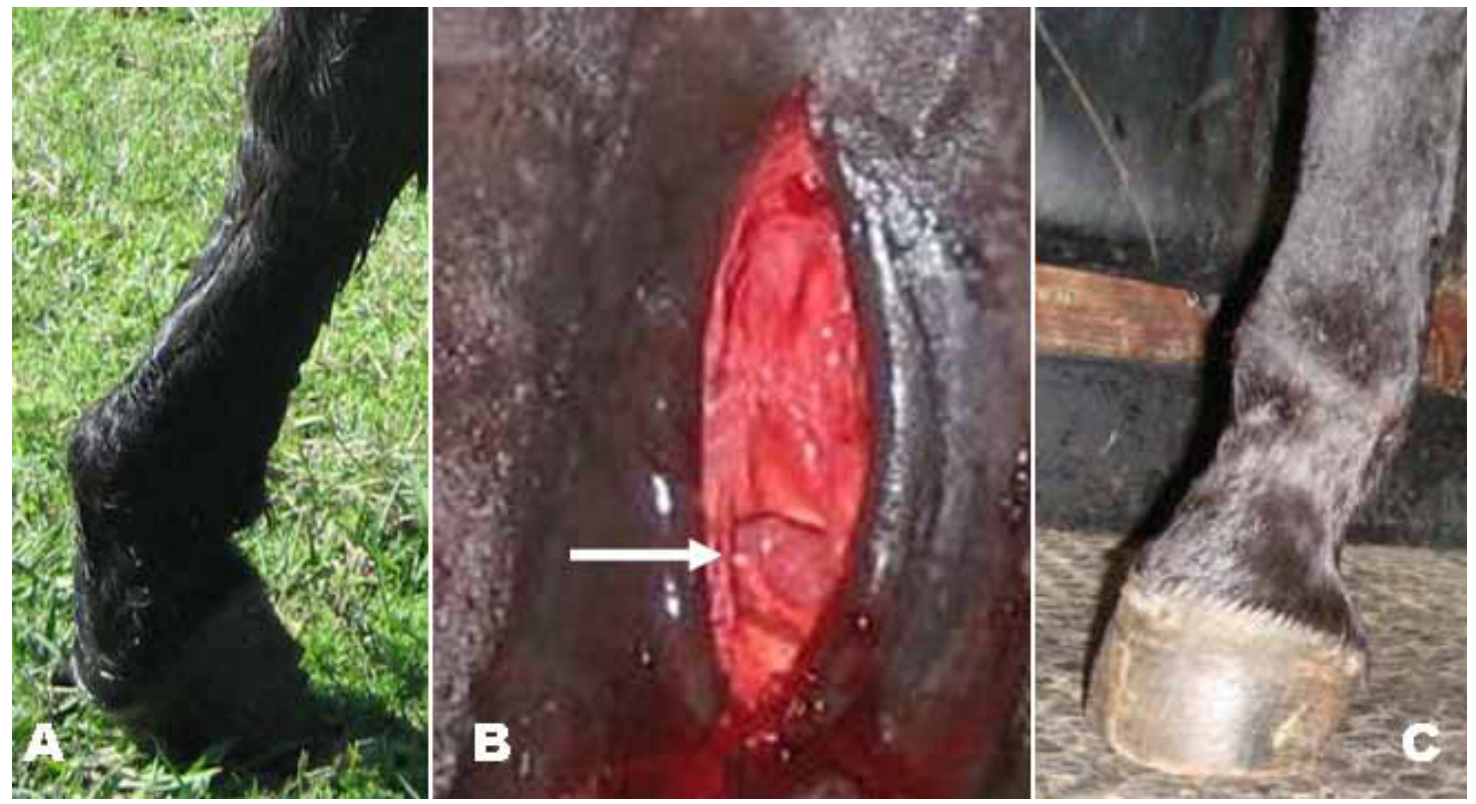

Figura 1. [A] Deformidade flexural adquirida de grau II da articulação interfalangeana distal do MAE de um equino. [B] Local da secção do tendão flexor digital profundo em um equino (seta). [C] Retorno da configuração normal do MAE após tenotomia do TFDP em um equino.

resultar em contratura muscular [11]. Por se tratar de um animal com histórico de ter sido usado para tração, a causa provável da deformidade foi tanto a traumática quanto a dolorosa, sendo que ambas estão relacionadas diretamente.

A deformação flexora da articulação interfalangeana distal, também denominada contratura do TFDP, manifesta-se clinicamente pela elevação dos talões, projeção cranial da muralha e apoio da região cranial das articulações interfalangeanas no solo. [13]. No presente estudo, o animal apresentava crescimento exagerado do casco, o que o impossibilitava de apoiar com a sola, assim, apoiava com a parede dorsal do casco e consequentemente havia desgaste nessa região.

As deformidades que ocorrem na articulação interfalangeana distal podem ser classificadas em dois estágios; o estágio I ocorre quando o ângulo descrito entre a parede dorsal do casco e o solo não ultrapassa $90^{\circ}$, enquanto que no estágio II de contração, a relação entre a parede dorsal do casco e o solo ultrapassa a vertical $\left(>90^{\circ}\right)[2,7]$. No caso em questão, trata-se de uma contratura de estágio II, pois o animal pisava no solo com a parede dorsal do casco.

O nível de gravidade do quadro clínico é que determina a escolha da técnica cirúrgica para o tratamento nos casos de deformidades flexurais. Nos casos em que a angulação entre a parede do casco e do solo ultrapassa os $115^{\circ}$, recomenda-se a tenotomia do TFDP $[1,4]$. A técnica cirúrgica escolhida para o tratamento da deformidade neste caso foi a tenotomia do TFDP, pelo fato de a angulação entre o casco e o solo ser maior que $115^{\circ}$.

A cirurgia foi realizada com o animal em estação, concordando com a literatura consultada [10]. A técnica mostrou-se simples o suficiente para ser realizada com o animal em estação. Não houve complicações anestésicas nem cirúrgicas durante todo o procedimento, garantindo sua finalização com sucesso.

Existem dois acessos descritos para a realização da técnica cirúrgica, sendo que o primeiro se localiza na região palmar central da quartela e o segundo, na região distal logo após a bifurcação do tendão flexor digital superficial [5]. Neste caso, foi usado o segundo acesso cirúrgico citado acima, e não houve dificuldades para a localização, dissecção e transecção do TFDP.

O prognóstico para essas situações em animais adultos é reservado, pois muitos dos animais apresentam recidivas após alguns meses [6]. Neste caso, o tratamento estipulado para a deformidade flexural adquirida na articulação interfalangeana distal mostrou ser efetivo, pelo fato de o animal ter retornado a conformação normal do membro. Na reavaliação, após seis meses, não houve sinais de recidiva, garantindo uma melhora significativa da sua qualidade de vida.

\section{NOTAS INFORMATIVAS}

${ }^{1}$ Sedomin ${ }^{\circledR}$. Laboratórios König S.A. Avellaneda, Buenos Aires, Argentina.

${ }^{2}$ Xylestesin ${ }^{\circledR}$ 2\%. Cristália, Itapira, SP, Brasil.

${ }^{3}$ Artridine ${ }^{\circledR}$. Virbac. Hortolândia, SP, Brasil. 


\section{REFERÊNCIAS}

1 Adams S.B. \& Santschi E.M. 2000. Management of congenital and acquired flexural limb deformities. In: Proceedings of the 46th American Association of Equine Practitioners Annual Convention. (Texas, E.U.A). pp.119-125.

2 Auer J.A. 1992. Flexural deformities. In: Equine Surgery. Philadelphia: WB Saunders Company, pp.957-971.

3 Beeman G.M. 1990. Factors that may influence club feet: genetics, nutrition, stance and proper trimming and medical treatment. Equine Athlete. 3(1): 9.

4 Bohanon T.C. 2005. Angular and flexural limb deformities. In: Proceeding of the North American Veterinary Conference (Orlando, Florida). pp.123-126.

5 Fackelman C.G., Auer J.A., Orsini J. \& von Salis B. 1983. Surgical treatment of severe flexural deformities of the distal interphalangeal joint in young horses. Journal of the American Veterinary Medical Association. 182(9): 949.

6 Greet T.R.C. 2000. Managing flexural and angular limb deformities: the newmarket perspective. In: Proceedings of the 46th American Association of Equine Practitioners Annual Convention (Texas, E.U.A). pp.133-135.

7 Mcllwraitc C.W. \& Fessler J.F. 1978. Evaluation of inferior check ligament desmotomy for treatment of acquired flexor tendon contracture in the horse. Journal of the American Veterinary Medical Association. 294(3): 293.

8 Mcllwraitc C.W. \& James L.F. 1982. Limb deformities in foals associated with ingestion of locoweed by mares. Journal of the American Veterinary Medical Association. 181(3): 255.

9 Owen J. M. 1975. Abnormal flexion of the corono-pedal joint or "contracted tendons" in unweaned foals. Equine Veterinary Journal. 7(1): 40.

10 Redden R.F. 1988. Complicated laminitis. Proceedings of the 46th American Association of Equine Practitioners Annual Convention (San Diego, E.U.A). 34: 311.

11 Rooney J.R. 1966. Contracted foals. Cornell Veterinarian. 56(2): 173.

12 Stashak T.S. 2003. Adams: claudicacion en el cavallo. 5.ed. Buenos Aires: Inter-Médica, pp.637-645.

13 Thomassian A. 1996. Deformidades flexurais dos membros. In: Enfermidades dos cavalos. 3.ed. São Paulo: Varela, pp.165-179. 